\title{
Tracklet-Global Track Fusion Using Support Degree Function in Sensor Networks
}

\author{
Xiaobin $\mathrm{Li}^{1,2 *}$, En Fan ${ }^{3}$, Changhong Yuan ${ }^{4}$ and Pengfei $\mathrm{Li}^{4}$ \\ ${ }^{1}$ College of Software Engineering, Lanzhou Institute of Technology, Lanzhou \\ 730050, China \\ ${ }^{2}$ Jožef Stefan International Postgraduate School, Ljubljana 1000, Slovenia \\ ${ }^{3}$ Department of Computer Science and Engineering, Shaoxing University, \\ Shaoxing 312000, China \\ ${ }^{4}$ Air Defense Forces Academy, Zhengzhou 450052, China \\ xiaobin_li@foxmail.com
}

\begin{abstract}
For the situation with unknown qualities of local tracks in sensor networks, a new tracklet-global track fusion method using the support degree function (SDF-T2GTF) is proposed. According to the characteristic of actual transmission modes, two local estimates of a moving target in adjacent interval transmitted by the same local node are defined as a tracklet, and subsequently tracklet-global track (T2GT) fusion can replace the traditional track fusion in the global node, namely local track-global track (LT2GT) fusion. Considered the advantage of the fuzzy track association (TA) method for unknown prior information of local tracks, it is used in T2GT association. Then all correlated tracklets in the same interval can be mapped into a set of points in parameter space by the Hough transform (HT) algorithm. The support degree function of these points is utilized to dynamically estimate the qualities of tracklets and reasonably allocates the weights of local estimates in fusion results. Hence, the proposed method can realize T2GT fusion without the prior information of local tracks. The experimental result illustrates that the proposed method can satisfy the requirement of data transmission in real systems, and can realize T2GT fusion; on the other, it can improve the performance of track fusion in accuracy compared with the traditional methods.
\end{abstract}

Keywords: Multiple Target tracking; Track Fusion; Tracklet; Hough Transform; Support Degree Function

\section{Introduction}

In sensor networks, information fusion integrates various information in different sources, patterns and forms observed by different sensors or multiple sources, and then utilize the redundancy and complementary between different information to realize the optimization and combination. Consequently, it can obtain better performance of fusion results in quantity and quality, and generate a consistent interpretation or presentation for observed environments [1-2]. Multiple target tracking (MTT) is at the position level of information fusion in sensor networks [3-5]. According to the data processing in fusion systems, MTT can be mainly divided into three phases: track initialization (or called measurement-measurement association), measurement-track association/fusion and tracktrack association/fusion. Track fusion is also a difficult problem in MTT [6-8]. Its aim is to keep the consistence of tracking results, generate the global estimates and form the

${ }^{*}$ Corresponding Author 
uniform situations. Its key issue is how to use the redundant and complement information included in the estimates of local tracks.

The traditional track fusion methods mainly consider the relative problems with various noises, deviations and uncertainties [9]. Unfortunately, the prior information required is difficultly obtained in real applications. A lot of works have been made for track fusion, particularly in the situation with unknown qualities of local tracks. To solve the problem of track association (TA) and track fusion in distributed multisensormultitarget multiple-attribute environments, a fuzzy track fusion method proposed in [10] directly utilizes the correlated local tracks observed by the most accurate sensor as the fusion results. It can avoid the decreased performance of fusion results when there exists a great difference of the sensors' performance. However, it brings less robustness and multisource of fusion results particularly in the situation that different sensors possess equivalent performances. In distributed target tracking systems, a track fusion algorithm based on track fuzzy membership developed in [11] use the fuzzy clustering method to calculate the membership degrees between local tracks to global tracks. To reduce fusion error for inflection points of tracks, an uncertainty analysis-based track fusion (UA-TF) method is presented in [12]. The UA-TF method can approaches a high accuracy with less computational burden and gain a tradeoff between accuracy and computational burden. A weighted track fusion method based on weighting factors dynamic allocation proposed in [13] exploits track information on the same target from multiradar systems to online estimate the accuracies of all tracks from all radars. A distributed multisensor track fusion method based dynamic weight (DW-DMSTF) in [14] defines a fuzzy support degree function of the estimates of local tracks to estimate their weights in fusion results. Nevertheless, most the proposed methods above only consider the effects of the current estimates of local tracks on track fusion, and ignore the impacts of the history estimates. For this reason, Gao et. al., in [15] map correlated tracks into a set of points in highdimensional space and utilizes the fuzzy clustering method to solve the problem of track fusion. Because correlated objects are the whole tracks, it increases the updating time of global tracks and difficultly satisfies the requirement of real-timely data processing. Furthermore, Fan et. al., in [16] present a tracklet-global track (T2GT) association according to the characteristics of transmission mode in real tracking systems, and it provides a good idea for track fusion. In addition, the messages transmitted between two nodes don't include the covariances of local estimates generally due to limited bandwidths in battlefields, and it leads to the constraints of the traditional track fusion methods directly applied in real application.

Normally, the track fusion methods designed are not only related with the types of sensors, but also with the fusion structure of tracking systems and the transmission modes of messages. Considered modeling errors in tracking systems and random disturbances from real environments, the qualities of local tracks are difficult to directly determine by the performance of sensors. In the situation with unknown prior information of local tracks, one can only utilize the information hided in local tracks to estimate their qualities [14]. Therefore, a T2GT fusion method using the support degree function (SDF-T2GTF) is proposed based on [14] and [16]. In the proposed method, a tracklet is defined as two local estimates of a moving target in adjacent interval transmitted by the same local node, and then local track-global track (LT2GT) fusion is real-timely divided into T2GT fusion. After LT2GT by using the fuzzy TA method [17], all the tracklets in the same interval can be transformed as a set of points in parametric space by the Hough transform (HT) method. Furthermore, the support degree function on these points is used to evaluate the qualities of tracklets, which act as the weights of local estimates in fusion results, and T2GT fusion can be realized by the sums of weighted local estimates. Finally, a simulation experiment is utilized to illustrate the validity of the proposed method. 


\section{Data Processing in Sensor Networks}

Generally, there exist two types of structures on track fusion in tracking systems [18]: local track-local track (LT2LT) fusion and local track-global track (LT2GF) fusion, as shown in Fig. 1. Because the former has no full utilization of the prior information on the estimates of local tracks, its performance is not as good as that of the latter. Consequently, this paper mainly discusses LT2GT fusion. Due to the restraint of communication bandwidth in battlefields, the estimates of local tracks are orderly transmitted from local nodes to the global node (or called the fusion center) by message. Moreover, each message only contains single estimate of local tracks. Hence, two types of tracks are generated in the global node: global tracks utilized for target tracking, and tracklets used for updating global tracks [16-19]. In [16], a tracklet is defined as two state estimates of a local track in continuous times, namely

$\boldsymbol{t}_{l, k}^{i}=\left\{\hat{\boldsymbol{x}}_{l, k-1}^{i}, \hat{\boldsymbol{x}}_{l, k}^{i}\right\}$

where, $\hat{\boldsymbol{x}}_{l, k-1}^{i}$ and $\hat{\boldsymbol{x}}_{l, k}^{i}$ are the estimates of the local track $T_{l}^{i}$ at time $k-1$ and $k$, respectively. In addition, a candidate tracklet is defined as single estimate of a local track as follow:

$\boldsymbol{t}_{l, k}^{i}=\left\{\hat{\boldsymbol{x}}_{l, k}^{i}\right\}$

Based on the above facts, T2GT fusion is proposed to replace LT2GT fusion.

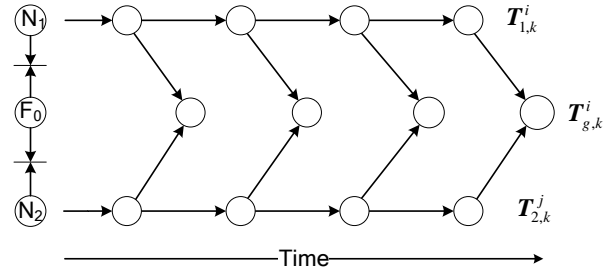

(a) Local Track-Local Track Fusion

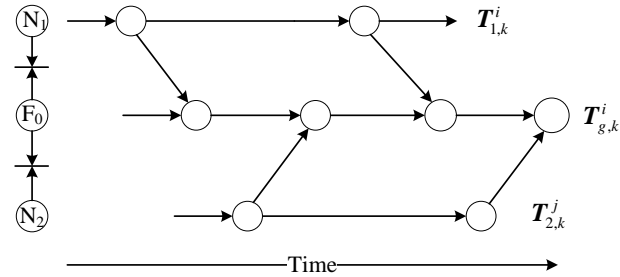

(b) Local Track-Global Track Fusion

Figure 1. Structures of Track Fusion

Generally, the trajectory of a target stays moving for a long time, and it can be regarded as non-linear [17]. Comparatively, because a tracklet keeps moving for a short time and has small change rate in state estimates, then it can be regarded as linear. Hence, all the tracklets in the same time interval can be seemed as a sequence of straight lines in the plane, and they can be mapped as a set of points in parameter space. As a result, the problem of track fusion can be transform to solve in parameter space. The concrete procedures are given as follows:

1) Map the tracklet $\boldsymbol{t}_{l, k}^{i}$ as the point $\boldsymbol{p}_{l, k}^{i}$ in parameter space:

$\boldsymbol{p}_{l, k}^{i}=f\left(\boldsymbol{t}_{l, k}^{i}\right)=\left(\rho_{l, k}^{i}, \theta_{l, k}^{i}\right)$

where $f(\cdot)$ is the mapping function from the tracklet $\boldsymbol{t}_{l, k}^{i}$ to the point $\boldsymbol{p}_{l, k}^{i} ; \hat{x}_{l, k}^{i}$ and $\hat{y}_{l, k}^{i}$ are the components of $\hat{\boldsymbol{x}}_{l, k}^{i}$ in x-axis and y-axis direction respectively; $\rho_{l, k}^{i}$ and $\theta_{l, k}^{i}$ are the coordinates of the corresponding point by transforming the estimate $\hat{\boldsymbol{x}}_{l, k}^{i}$ into parameter space, namely

$\rho_{l, k}^{i}=\left|\eta_{l}^{i} \hat{x}_{l, k}^{i}-\hat{y}_{l, k}^{i}\right| / \sqrt{\left(\eta_{l}^{i}\right)^{2}+1}$ 
$\theta_{l, k}^{i}=\arctan \left(-1 / \eta_{l}^{i}\right)$

$\eta_{l}^{i}=\left(\hat{y}_{l, k}^{i}-\hat{y}_{l, k-1}^{i}\right) /\left(\hat{x}_{l, k-1}^{i}-\hat{x}_{l, k-1}^{i}\right)$

2) Establish the set of points corresponding to the sequence of tracklets

$\left\{\boldsymbol{p}_{l, k}^{i} \mid\left(\rho_{l, k}^{i}, \theta_{l, k}^{i}\right)=f\left(\boldsymbol{t}_{l, k}^{i}\right), i=1,2, \mathrm{~L}, m\right\}$

Where $M$ is the number of tracklets in local nodes.

\section{Traditional Fuzzy TA Method}

Considering the advantage of the fuzzy TA method, a synthetic function is selected to calculate the association degrees between tracklets to local tracks [2]. The fuzzy TA method is mainly divided into three steps:

1) Determine the fuzzy factor set:

In real applications, the position difference, velocity difference and heading difference in horizontal direction are usually utilized as three factors in association decision. Expressed that $\hat{\boldsymbol{x}}_{l, k}^{i}=\left[x_{l, k}^{i}, \hat{\dot{x}}_{l, k}^{i}, y_{l, k}^{i}, \hat{\dot{y}}_{l, k}^{i}\right]^{\mathrm{T}}$ is the current estimate of the tracklet $\boldsymbol{t}_{l, k}^{i}$, and that $\hat{\boldsymbol{x}}_{g, k \mid k-1}^{j}=\left[x_{g, k \mid k-1}^{j}, \hat{\dot{x}}_{g, k \mid k-1}^{j}, y_{g, k \mid k-1}^{j}, \hat{\dot{y}}_{g, k \mid k-1}^{j}\right]^{\mathrm{T}}$ is the predict vector of the local track $T_{g, k-1}^{j}$, then the position difference, velocity difference and heading difference can be respectively calculated by:

$$
\begin{aligned}
& u_{1}=\sqrt{\left(\hat{x}_{l, k}^{i}-\hat{x}_{g, k \mid k-1}^{j}\right)^{2}+\left(\hat{y}_{l, k}^{i}-\hat{y}_{g, k \mid k-1}^{j}\right)^{2}} \\
& u_{2}=\left|\sqrt{\hat{\dot{x}}_{l, k}^{i}{ }^{2}+\hat{\dot{y}}_{l, k}^{i}{ }^{2}}-\sqrt{\hat{\dot{x}}_{g, k \mid k-1}^{j}{ }^{2}+\hat{\dot{y}}_{g, k \mid k-1}^{j}}\right| \\
& u_{3}=\mid \arctan \frac{\hat{\dot{y}}_{l, k}^{i}}{\hat{\dot{x}}_{l, k}^{i}-\arctan \frac{\hat{\dot{y}}_{g, k \mid k-1}^{j}}{\hat{\dot{x}}_{g, k \mid k-1}^{j}} \mid}
\end{aligned}
$$

Hence, the fuzzy factor set may be expressed as $\boldsymbol{U}=\left\{u_{1}, u_{2}, u_{3}\right\}$.

\section{2) Select the synthetic function}

Based on the above fact, the similarity vector can be expressed by the Gaussian membership function:

$$
D_{i j}(3)=\left[d_{i j}\left(u_{1}\right), d_{i j}\left(u_{2}\right), d_{i j}\left(u_{3}\right)\right]^{\mathrm{T}}=\left[\exp \left(-\frac{u_{1}}{u_{1 \max }}\right), \exp \left(-\frac{u_{2}}{u_{2 \max }}\right), \exp \left(-\frac{u_{3}}{u_{3 \max }}\right)\right]^{\mathrm{T}}
$$

Here, $d_{i j}(\cdot)$ is the similarity measure, $u_{1 \max }, u_{2 \max }$ and $u_{3 \max }$ are the corresponding maximum values of the position error, velocity error and heading error in horizontal direction, which are the empirical values.

\section{3) Calculate the association degree}

Due to the existence of many synthetic functions, one utilizes the following synthetic function

$$
f\left(D_{i j}(r)\right)=\sum_{l=1}^{r} a_{l} d_{i j}\left(u_{l}\right), \quad a_{l} \in[0,1], \sum_{l=1}^{r} a_{l}=1
$$


as the decision function for association decision. Considering the influence of different fuzzy factors on association decision, one can set $a_{1}=0.55, a_{2}=0.35$ and $a_{3}=0.1$.

Based on Eq. (12), the fuzzy association degree between the tracklet $\boldsymbol{t}_{l, k}^{i}$ and global track $T_{g, k}^{j}$ can be calculated by:

$\mu_{i j}\left(t_{l, k}^{i}, T_{g, k-1}^{j}\right)=f\left(D_{i j}(3)\right)=\sum_{l=1}^{3} a_{l} d_{i j}\left(u_{l}\right)$

Here, $\mu_{i j}\left(\boldsymbol{t}_{l, k}^{i}, T_{g, k-1}^{j}\right)$ is the weighted result by $d_{i j}\left(u_{1}\right), d_{i j}\left(u_{2}\right)$ and $d_{i j}\left(u_{3}\right)$, which are three components of the synthetic similarity degree. After the calculation of fuzzy association degrees between a tracklet and all the global tracks, then the tracklets with the maximum association degree are determined to correlate with the global track.

\section{T2GT Fusion Method Based on Support Degree Function}

Because local tracks from different local nodes possess different qualities, the corresponding tracklets has unequal weights in fusion results. Based on this fact, if tracklets are directly utilized into track fusion, it will lead to degrading the performance of fusion results in accuracy. Considered modeling error in tracking systems and random disturbances from real environments, the qualities of tracklets can be determined according to the information hided in local estimates. Due to the establishment of velocity and accelerated velocity on position estimates, the effects of position estimates on filtered results are superior to them [14]. Moreover, tracklets consist of two estimates, which provide more information compared with single estimate, and then it can reduce the uncertainty of track fusion [16]. Therefore, by mapping tracklets as a set of points, the proposed method utilizes the support degree function on these points to realize T2GT fusion.

\subsection{Support Degree Function of Tracklets}

In parametric space, the distance of two mapped points can be defined as:

$d_{i j}=\left|\boldsymbol{p}_{l, k}^{i}-\boldsymbol{p}_{g, k}^{j}\right|$

where the point $\boldsymbol{p}_{l, k}^{i}$ is the mapped point of the tracklet $\boldsymbol{t}_{l, k}^{i}$ in parametric space; $\boldsymbol{p}_{g, k}^{j}$ is that of the combination consisted of the estimate $\hat{\boldsymbol{x}}_{g, k-1}^{j}$ of the global track $T_{g, k}^{j}$ at time $k-1$ and the predicted position $\hat{\boldsymbol{x}}_{g}^{j}(k \mid k-1)$ at time $k$, namely

$\boldsymbol{p}_{g, k}^{j}=f\left(\left[\hat{\boldsymbol{x}}_{g, k-1}^{j}, \hat{\boldsymbol{x}}_{g, k \mid k-1}^{j}\right]^{\mathrm{T}}\right)$

The corresponding predicted equation can be expressed as:

$\hat{\boldsymbol{x}}_{g, k \mid k-1}^{j}=F_{k-1} \hat{\boldsymbol{x}}_{g, k-1}^{j}$

Here, $F_{k-1}$ is the state-transition matrix at time $k-1$.

As known in Eq. (14), the value of the distance $d_{i j}$ becomes larger with the increase of the difference between $\boldsymbol{t}_{l, k}^{i}$ and $\boldsymbol{t}_{g, k}^{j}$, and then it shows the relative support degree of these two tracklets is smaller. Because one only utilizes the definition of the distance is only based on the mapped points, and it can relax the requirement of priori information on local tracks. Based on this fact, one can define the support degree function as follow:

$r_{i j}=-\frac{d_{i j}}{\max \left\{d_{i j}\right\}}+1$ 
Here, $d_{i j}>0, r_{i j} \in[0,1], \max \left\{d_{i j}\right\}$ is the maximum value in all the distances for any two points. Based on Eq. (17), $r_{i j}$ is in inverse proportion with $d_{i j}$. Namely, the support degree function of tracklets decreases with the increased distance between the corresponding mapped points; on the contrary, the support degree function of tracklets increases with the decreased distance between the corresponding mapped points. Therefore, the support degree function possesses the advantage of fuzzy membership functions to avoid the absolution of support degrees on tracklets.

\subsection{T2GT Fusion Method}

From the facts in Section 4.1, $r_{i j}$ only expresses the mutual support degree of two tracklets $\left(\boldsymbol{t}_{l, k}^{i}\right.$ and $\left.\boldsymbol{t}_{g, k}^{j}\right)$, and it can't reflect the support degree of the tracklet $\boldsymbol{t}_{l, k}^{i}$ relative to all the other tracklets at time $k$. For this reason, $r_{i j}$ needs to be further normalized as follow:

$\omega_{i j}=\frac{r_{i j}}{r_{1 j}+r_{2 j}+\cdots+r_{m j}}$

Here, if $\omega_{i j}$ becomes increasingly great, it indicates the tracklet $\boldsymbol{t}_{l, k}^{i}$ relative to all the tracklets are increasingly high, namely the local estimate $\hat{\boldsymbol{x}}_{l, k}^{i}$ is more reliable.

Based on the above analysis, the global estimate $\hat{\boldsymbol{x}}_{g, k}^{j}$ (or called the fusion result) of the global track $T_{g, k}^{j}$ at time $\mathrm{k}$ can be expressed by

$\hat{\boldsymbol{x}}_{g, k}^{j}=\omega_{1 j} \hat{\boldsymbol{x}}_{1, k}^{(1)}+\omega_{2 j} \hat{\boldsymbol{x}}_{2, k}^{(2)}+\cdots+\omega_{m j} \hat{\boldsymbol{x}}_{m, k}^{(m)}$

where $m$ is the number of tracklets correlated with the global track $T_{g, k}^{j}$.

\section{Experiment Results and Analysis}

To evaluate the performance of the proposed method in comparison with the DWDMSTF method in [13], a simulation experiment is employed as follow. In addition, 100 Monte Carlo runs by MATLAB 2009a version software have been performed by using a computer with a dual-core CPU of Pentium $42.93 \mathrm{GHz}, 1-\mathrm{GB}$ RAM.

In the simulation scenario, there exist four crossing targets, moving at constant velocity and keeping the height with $1.0 \mathrm{~km}$, as shown in Figure 2. Their motion model and observation model are defined as follows:

$$
\begin{aligned}
\boldsymbol{x}_{i, k} & =\left[\begin{array}{cccc}
1 & \frac{\sin \omega_{i} T}{\omega_{i}} & 0 & \frac{1-\cos \omega_{i} T}{\omega_{i}} \\
0 & \cos \omega_{i} T & 0 & -\sin \omega_{i} T \\
0 & \frac{1-\cos \omega_{i} T}{\omega_{i}} & 1 & \frac{\sin \omega_{i} T}{\omega_{i}} \\
0 & \sin \omega_{i} T & 1 & \cos \omega_{i} T
\end{array}\right] \boldsymbol{x}_{i, k-1}+\boldsymbol{v}_{i, k} \\
\boldsymbol{z}_{j, k} & =H \boldsymbol{x}_{i, k}+\boldsymbol{w}_{i, k}
\end{aligned}
$$

Here, $\boldsymbol{x}_{i, k}$ is an $n$-dimensional state vector, $\boldsymbol{z}_{j, k}$ is an $m$-dimensional observation vector; $H$ is an $m \times n$ observation transition matrix; $\omega$ is the turn rate; the process noise $v_{i, k}$ and the observation vector $\boldsymbol{w}_{i, k}$ are assumed to the zero-mean Gaussian noises with the 
covariance $Q_{k}$ and the covariance $R_{k}$, respectively. Their initial states are given by $(9.0 \mathrm{~km}, 200 \mathrm{~m} / \mathrm{s}, 9.5 \mathrm{~km}, 200 \mathrm{~m} / \mathrm{s})^{\mathrm{T}},(9.0 \mathrm{~km}, 200 \mathrm{~m} / \mathrm{s}, 7.5 \mathrm{~m}, 200 \mathrm{~m} / \mathrm{s})^{\mathrm{T}}, \quad(9.0 \mathrm{~km}, 200 \mathrm{~m} / \mathrm{s}$, $5.5 \mathrm{~km}, 200 \mathrm{~m} / \mathrm{s})^{\mathrm{T}}$, and $(9.0 \mathrm{~km}, 200 \mathrm{~m} / \mathrm{s}, 18.0 \mathrm{~km},-200 \mathrm{~m} / \mathrm{s})^{\mathrm{T}}$, respectively. In the distributed sensor network, there is a global node $\mathrm{F}_{0}$ and four radars $\left(\mathrm{N}_{i}, i=1,2,3,4\right)$. Here, the position of the global node is located at $(21.0 \mathrm{~km}, 24.0 \mathrm{~km})$, and four radars are assumed to observe targets synchronously with the sampling interval $T=2 \mathrm{~s}$. In addition, the positions and performances of four radars are provided by Tab. 1. The steps of data processing in the system is as follows: 1) generate local tracks by the collected measurements in local nodes; 2) transform the state estimates of local tracks from polar coordinate system to global rectangular coordinate system; 3) transmit these estimates orderly to the global node; 4) generates tracklets in the global node; 5) utilize the fuzzy TA method mentioned above and the proposed method to respectively realize the association and fusion between tracklets and global tracks. The measurements of four targets obtained by four radars are shown as Figure 2. Figure 3, shows the fusion results by using the proposed method. For analyzing its performance of fusion results, Figure 4, shows their position root mean-squared errors (RMSE) for four targets by using two track fusion methods. From Figure 4, the position RMSE by the proposed method is smaller than those by the DW-DMSTF method. Hence, it certifies the validity of the proposed method.

Table 1. Positions and Performances of Four Radars

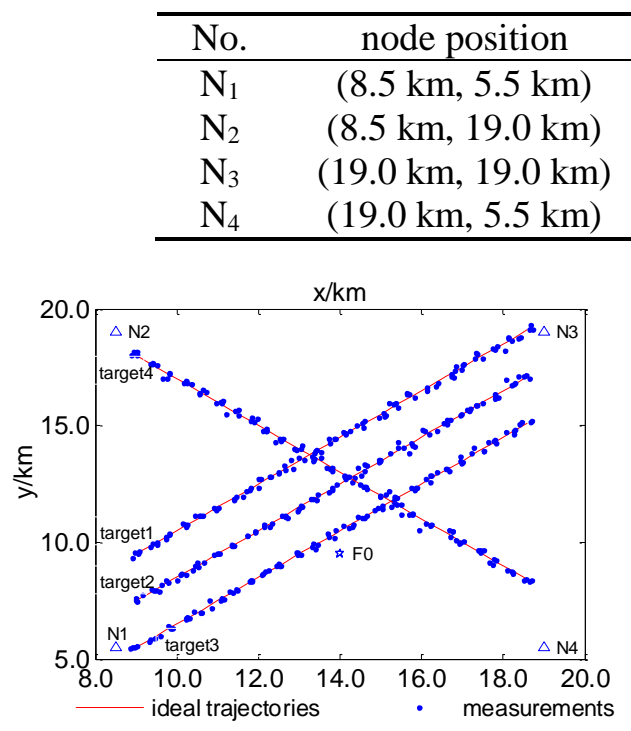

Figure 2. Ideal Trajectories

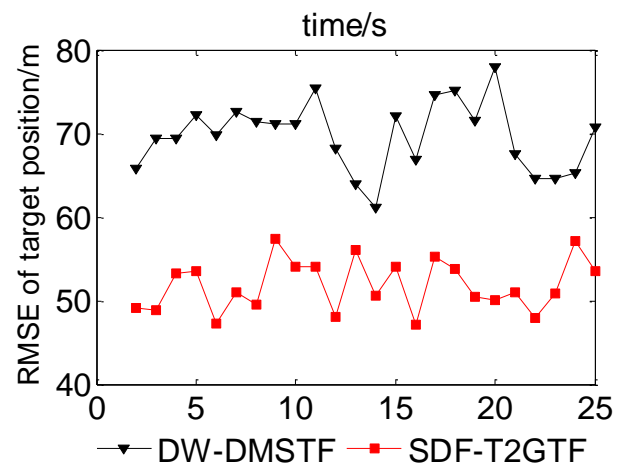

(a) Target 1

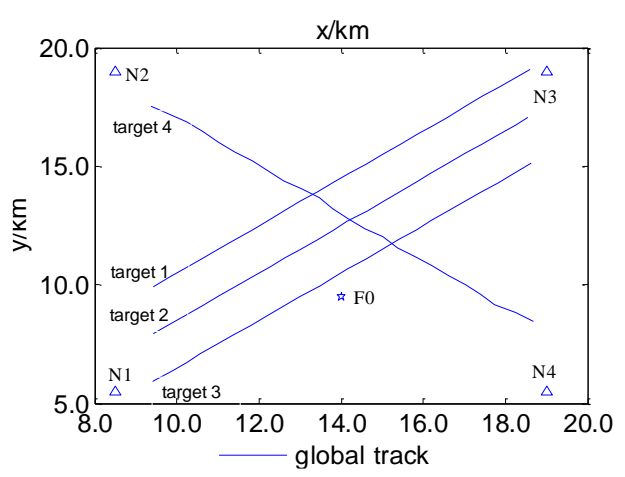

Figure 3. Fusion Results

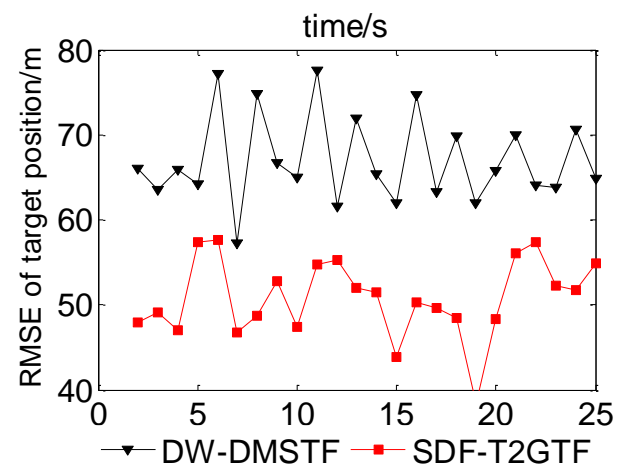

(b) Target 2 


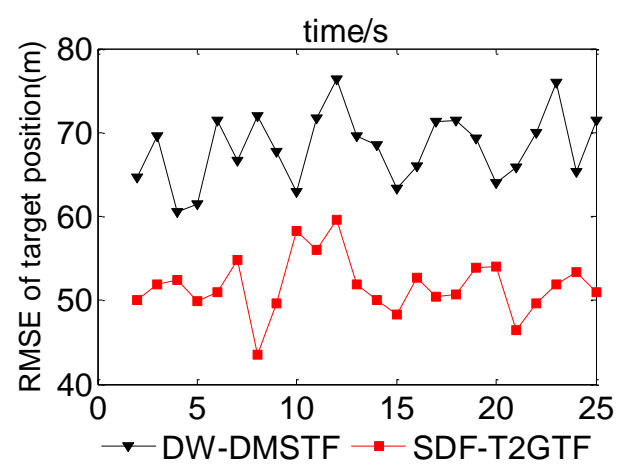

(c) Target 3

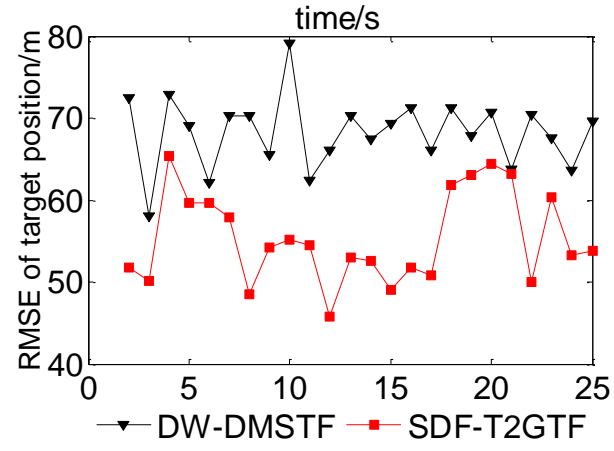

(d) Target 4

Figure 4. Tracking Results by Two Track Fusion Methods

\section{Conclusion}

Track fusion is a difficult problem on MTT in sensor networks. For real applications of track fusion, this paper firstly analyzes the shortcomings of the traditional track fusion methods. In the situation with unknown qualities of local tracks, a T2GT fusion method using the support degree function is proposed. In the proposed method, the definition of tracklets is given according to the characteristics of transmission mode in real tracking systems, and then LT2GT fusion can be divided into T2GT fusion in real time. Then the problem of track fusion can be transformed to solve in parametric space by the HT method. Through dynamic estimation of the qualities of tracklets using the support degree function, the weights of the current estimates can be reasonably allocated in fusion results to realize T2GT fusion. The simulation experiment illustrates the proposed method can meet the transmission requirement in real tracking systems and meanwhile keep the robust of fusion results. Furthermore, it can improve the accuracy of fusion results compared with the DW-DMST fusion method.

\section{Acknowledgments}

This work is supported by the National Natural Science Foundation of China (No. 61301074), the Startup Project of Doctor Science Research of Shaoxing University (No. 20155015), Science Project of Shaoxing University (No. 2015LG1006), Natural Science Foundation of Shenzhen Polytechnic (No. 601522K21017), and Lanzhou Science and Technology Project (No. 2015-4-3).

\section{References}

[1] B. Khaleghi, A. Khamis, and F. O. Karry, "Multisensor data fusion: A review of state-of-the-art", Information Fusion, vol. 14, (2013), pp. 28-44.

[2] Y. He, G. X. Wang, X. Guan, "Information fusion theory with applications", Publishing House of Electronics Industry, Beijing, (2010).

[3] W. Zhang, Y. Duan and L. Zhou, "New fuzzy optimal assignment algorithm of distributed multi-sensor multi-target tracking system", Journal of Computational Information Systems, vol. 9, (2013), pp. 55935600.

[4] F. H. Jin and H. P. Wang, "Flight tracking algorithm of FFSR based on fuzzy logic", Journal of Harbin University of Science and Technology, vol. 18, no. 4, (2013), pp. 98-102.

[5] L. Qiao, "Research of passive tracking arithmetic based on spatial information measurement", Journal of Harbin University of Science and Technology, vol. 17, no. 4, (2012), pp. 64-67.

[6] E. Fan, W. X. Xie and Z. X. Liu, "Maneuvering target tracking using fuzzy logic-based recursive least squares filter", Eurasip Journal on Advances in Signal Processing, vol. 2014, (2014), pp. 1-9.

[7] E. Fan, W. X. Xie, Z. X. Liu and P.F. Li, "Combining generalized JPDA and FRLS filter for tracking multiple maneuvering targets", The 12th IEEE International Conference on Signal Processing (ICSP'14), Hangzhou, China, (2014), pp. 239-245.

[8] E. Fan, W. X. Xie and Z. X. Liu, "Reliability-weighted nearest neighbor track association in sensor network", Sensor Letters, vol. 12, (2014), pp. 319-324. 
[9] K. C. Chang, R. K. Saha and Y. Bar-Shalom, “On optimal track-to-track fusion”, IEEE Transactions on Aerospace and Electronic Systems, vol. 33, (1997), pp. 1271-1276.

[10] A. M. Aziz, "Fuzzy track-to-track association and track fusion approach in distributed multisensormultitarget multiple-attribute environment", Signal Processing, vol. 87, (2007), pp. 1474-1492.

[11] J. H. Ran and Y. Zhang, "Distributed system data fusion algorithm based on track fuzzy membership", Journal of Signal Processing, vol. 27, (2011), pp. 226-229.

[12] L. Xu, P. J. Ma and X .T. Su, "An uncertainty analysis-based track track fusion algorithm", Journal of Astronautics, vol. 32, (2011), pp. 567-573.

[13] Y. P. Huang, Y. F. Zhou, H. B. Zhang and X. H. Tang, "Algorithm of weighting factors dynamic allocation in multi-radar track weighted fusion", Computer Applications, vol. 28, (2008), pp. 2452-2454.

[14] Z. T. Hu and X. X. Liu, "Distributed multi-sensor track fusion algorithm based on dynamic weight", Application Research of Computers, vol. 6, (2006), pp. 59-61.

[15] X. B. Gao and J. Li, "A feature-weighted fuzzy clustering algorithm for track association in sensor network", Acta Electronic Sinca, vol. 35, (2007), pp. 148-151.

[16] E. Fan, W. X. Xie and Z. X. Liu, "Fuzzy track association using tracklet-based Hough transform, Journal of Shenzhen University Science and Engineering", vol. 30, (2013), pp. 551-556.

[17] S. Mori, W. H. Barker, C. Y. Chong and K. C. Chang, "Track association and track fusion with nondeterministic target dynamics", IEEE Transactions on Aerospace and Electronic Systems, vol. 32, (2002), pp. 659-668.

[18] W. H. Yang, "Multisensor data fusion and its application", Publishing House of Xidian University, Xi'an, (2004).

[19] Q. G. Ge and J. L. Li, "Analysis of the principle for elimination of redundant information in distributed fusion”, Command Information System \& Technology, vol. 1, (2010), pp. 12-15.

\section{Author}

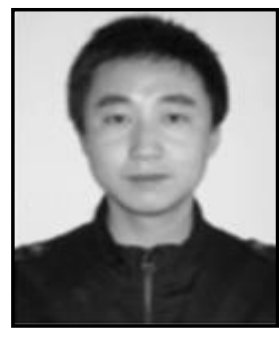

Xiaobin Li, received his Bachelor's Degree in Computer Science and Technology from Northwest Normal University, Lanzhou, China, in 2002, Master's Degree in Computer Science from Jožef Stefan International Postgraduate School, Ljubljana, Slovenia, in 2011. He is currently working in College of Software Engineering, Lanzhou Institute of Technology, Lanzhou, China and pursuing a doctoral degree in Jožef Stefan International Postgraduate School, Ljubljana, Slovenia. His fields of interests include fuzzy information processing, data fusion, multiple target tracking, sensor networks, software engineering. 
International Journal of Signal Processing, Image Processing and Pattern Recognition Vol. 9, No. 10, (2016) 